\title{
Nonlinear Outcome of Gravitational Instability in Optically Thick Disks
}

\author{
Charles F. Gammie \\ Center for Astrophysics, 60 Garden St., Cambridge, MA, USA
}

\begin{abstract}
.
In the outer parts of disks in active galactic nuclei and around young stellar objects (YSOs) the internal sources of heat may be too weak to prevent gravitational instability. We are thus motivated to examine the nonlinear outcome of gravitational instability in an optically thick disk. We use height-integrated, two-dimensional numerical simulations that include pressure (the gas has a polytropic equation of state), self-gravity, and cooling. The cooling function is calculated using a one-zone model and is characterized by a single dimensionless parameter $\tau_{\text {cool }}$, which is the ratio of the cooling time to the orbital period. The simulations are done in the context of a local model of the disk (the "shearing sheet"). We also include the possibility of an effective "viscosity" characterized by a dimensionless parameter $\alpha$.

We find that the gravitational instability in thin, Keplerian disks leads to sustained angular momentum transport that is essentially local in character. Instability drives $Q$ toward 1.7, and provides an angular momentum flux $\alpha_{\text {eff }} \simeq 0.4 / \tau_{\text {cool }}$. The disk breaks up when the cooling time is shorter than the dynamical time, which is equivalent to saying that $\alpha_{e f f}<1$. We conclude that gravitational instability can raise the angular momentum transport rate in the outer parts of AGN and YSO disks (although YSO disks may be too thick for our local approach to be strictly applicable). This can occur over a factor of a few in radius. The gravitationally dominated region is bounded on the inside by that radius where other heating processes (e.g. MHD turbulence driven by the Balbus-Hawley instability) make $Q>1$. It is bounded on the outside by that radius where the cooling time is shorter than the dynamical time, or the disk is optically thin so that the equation of state is soft. At larger radius the disk will be clumpy.
\end{abstract}

This work was supported under NAG 52837. 